\title{
Krüppel-Like Factor 4 Inhibits Pancreatic Cancer Epithelial-to-Mesenchymal Transition and Metastasis by Down- Regulating Caveolin-1 Expression
}

\author{
Zhonglin Zhu ${ }^{a} \quad$ Zhilong Yu ${ }^{a} \quad$ Jianfeng Wanga,b Lisheng Zhou ${ }^{a} \quad$ Jing Zhang ${ }^{a}$ \\ Bin Yao Jin Dou ${ }^{a}$ Zhengjun Qiu ${ }^{a}$ Chen Huanga \\ aDepartment of General Surgery, Shanghai General Hospital, Shanghai Jiaotong University School of \\ Medicine, Shanghai, 'Department of Gastrointestinal surgery, Jiangyin People's Hospital, Jiangsu, \\ P. R. China
}

\section{Key Words}

Klf4 • Caveolin-1 Pancreatic cancer $・$ Epithelial-to-mesenchymal transition • Metastasis

\begin{abstract}
Background/Aims: Krüppel-like factor 4 (KLF4), a member of the KLF family of zinc finger transcription factors, has been identified as a tumor suppressor gene in a variety of tumors. However, the molecular mechanisms by which KLF4 inhibits epithelial-to-mesenchymal transition (EMT) and metastasis in pancreatic cancer remain unclear. Methods: KLF4 expression in pancreatic cancer was analyzed using public datasets (Oncomine and The Cancer Genome Atlas). The expression of KLF4, caveolin-1 (Cav-1), E-cadherin, and vimentin, and their correlations with clinicopathological characteristics were evaluated by immunohistochemistry in pancreatic cancer tissues. The biological functions and underlying mechanisms of KLF4 expression on EMT and metastasis were also investigated in vitro and in vivo. Results: Public datasets showed that KLF4 expression was significantly decreased in pancreatic cancer and correlated with the depth of invasion and disease stage. The expression of KLF4, Cav-1, E-cadherin, and vimentin protein in pancreatic cancer tissues was closely associated with pathological grade, disease stage, and metastasis. KLF4 expression was also positively correlated with E-cadherin expression and negatively correlated with vimentin expression, whereas Cav- 1 expression was negatively associated with E-cadherin expression and positively correlated with vimentin expression. Knockdown of KLF4 expression promoted EMT and facilitated pancreatic cancer cell growth and metastasis in vitro and in vivo. In addition, immunohistochemistry (IHC) results indicated that KLF4 expression was negatively correlated with Cav-1 expression. Furthermore, down-regulating KLF4 expression increased Cav-1 and vimentin expression and decreased E-cadherin expression. Mechanistically, KLF4 could transcriptionally inhibit Cav-1 expression by binding directly to the promoter domain of Cav-1. Conclusions: KLF4 inhibits pancreatic Z. Zhu, Z. Yu, J. Wang and L. Zhou contributed equally to this work.
\end{abstract}


cancer EMT and metastasis by down-regulating Cav-1 expression, suggesting that the KLF4/ Cav-1 signaling pathway may be a novel diagnostic and therapeutic target.

(C) 2018 The Author(s)

Published by S. Karger AG, Basel

\section{Introduction}

Pancreatic cancer is the seventh leading cause of cancer-related deaths worldwide [1], and the third leading cause in developed countries, with an expected 53, 670 new diagnosed cases and 43, 090 deaths in 2016 in the USA alone [2]. In China, its incidence reached 90.1 per 100,000 people in 2015, with a mortality of approximately 79.4 per 100,000 people [3]. Although surgery may be curative for pancreatic cancer, only less than $20 \%$ of cases at presentation constitute suitable candidates for resection [4], with the 5-year survival rate being $<10 \%$ between 2005 and 2011 in the USA. Due to its early recurrence and metastasis, the long-term prognosis of patients who undergo surgical resection remains disappointing [5]. Accordingly, a deeper insight into the molecular mechanisms underlying the progression of pancreatic cancer is needed.

Krüppel-like factor 4 (KLF4), a transcription factor containing three successive $\mathrm{C} 2 \mathrm{H} 2$ zinc finger domains in its C-terminal [6], is expressed widely in mammals in the digestive system, skin, and oral cavity [7]. KLF4 participates in the transcriptional regulation of a variety of oncogenes, tumor suppressor genes, and multiple signaling pathways [8-10], which have been implicated in cell proliferation, differentiation, and apoptosis. Various studies have reported that KLF4 is involved in the development and progression of cancer, including breast cancer, gastric cancer, esophageal squamous cell carcinoma, osteosarcoma, and prostate cancer [1115]. Interestingly, KLF4, which contains both transactivation and transcriptional repression domains in its $\mathrm{N}$-terminal, can positively and negatively mediate tumor biology by regulating downstream target genes [16]. Recent studies have demonstrated that KLF4 expression is relatively low in pancreatic cancer tissues and cells compared with that in normal pancreatic tissues and cells, and have also revealed that decreased KLF4 expression is correlated with aggressive disease stages and metastasis $[17,18]$. In addition, KLF4 overexpression inhibits pancreatic cancer cell growth and metastasis in vitro and in vivo, whereas KLF4 knockdown has the opposite effect [19]. Co-expression of KLF4 and MSI2 or CD44 is remarkably correlated with pancreatic cancer proliferation and metastasis, suggesting that KLF4 may mechanistically cooperate with other factors $[20,21]$. These clinical and experimental findings indicate that KLF4 may act as a tumor suppressor gene in pancreatic cancer and is a potential target for therapeutic intervention. In breast cancer, KLF4 has been implicated in epithelial-to-mesenchymal transition (EMT) by increasing the expression of E-cadherin (an epithelial morphology biomarker), and decreasing the expression of vimentin and $\beta$-catenin (mesenchymal morphology biomarkers) [22]. Moreover, down-regulating KLF4 can increase the motility of cancer cells, indicating the fundamental roles of KLF4 in EMT and metastasis in breast cancer [22]. Nevertheless, the precise mechanisms underlying the role of KLF4 in pancreatic cancer EMT and metastasis are still poorly understood.

A great number of molecules have been implicated in the development and progression of pancreatic cancer, such as stat3 [23], FoxM1 [24], IL-1 $\beta$ and miR-29a [25]. Caveolin-1 (Cav-1), the major structural protein of plasma membrane invaginations [26], is a key signal transducer in various human cancers [27-29]. A growing body of evidence has shown that Cav-1 acts as an oncogene, promoting cancer development and progression [30, 31]. Further studies suggested that Cav-1 can promote EMT in pancreatic cancer by down-regulating epithelial markers and up-regulating mesenchymal markers [32]. In the present study, we determined the correlation between KLF4 expression and clinicopathological features and the regulating effects of KLF4 on Cav-1 expression and function. Our identification of a novel KLF4/Cav-1 signaling pathway regulating pancreatic cancer EMT and metastasis is expected to lead to the development of new diagnostic tools and therapeutic targets. 


\section{Cellular Physiology Cell Physiol Biochem 2018;46:238-252

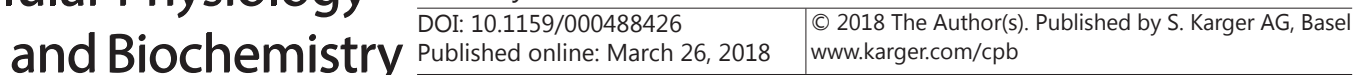 Zhu et al.: Krüppel-Like Factor 4 Inhibits Epithelial-to-Mesenchymal Transition and Metastasis}

\section{Materials and Methods}

\section{Cell lines and culture conditions}

The human pancreatic cancer cell line AsPC-1 was purchased from the American Type Culture Collection. AsPC- 1 cells were cultured at $37^{\circ} \mathrm{C}$ in Eagle's minimal essential medium supplemented with $10 \%$ fetal bovine serum, sodium pyruvate, nonessential amino acids, L-glutamine, and vitamin solution (Flow Laboratories).

\section{Human tissue microarray (TMA)}

The expression of KLF4, Cav-1, E-cadherin, and vimentin was analyzed using a TMA purchased from US Biomax, which included pancreatic cancer tissue samples from 70 cases and normal pancreatic tissue samples from 10 cases. US Biomax also provided information on the cases concerning sex, age, pathological diagnosis, clinical stage, histological grade, and so on. The use of tissue samples was in accordance with the relevant guidelines and regulations, and their use was approved by the Shanghai General Hospital Institutional Review Board.

\section{Western blot analysis}

Standard western blot analysis of whole-cell protein lysates was performed using primary antibodies against KLF4 (rabbit polyclonal; Santa Cruz Biotechnology), Cav-1 (mouse monoclonal; BD Biosciences), E-cadherin (mouse monoclonal; Santa Cruz Biotechnology), and vimentin (mouse monoclonal; Gene Tech Biotechnology), and a secondary anti-mouse or anti-rabbit IgG antibody (Amersham Life Sciences). Equal amounts of protein, which were blotted with an anti-GAPDH antibody (Santa Cruz Biotechnology), were used as loading controls.

\section{Plasmid construction and lentivirus transduction}

The full-length cDNA of KLF4 in the pcDNA3.1 vector (pKLF4) was purchased. A KLF4 shRNA lentivirus (shKLF4), with 5'-GACCAGGCACUACCGUAAAdTdT-3' (sense) and 3'-dTdTCUGGUCCGUGAUGGCAUUU-5' (anti-sense), was generated. pcDNA3.1 and control shRNA lentivirus were designed as the respective negative controls. pKLF4 and pcDNA3.1 were transfected into AsPC-1 cells with Lipofectamine 2000 CD (Invitrogen) before assays. In addition, cells treated with transfection reagent alone were used as mock transfection controls (mock). For lentivirus transduction, shKLF4, control shRNA lentivirus (control), or lentivirus (mock) were each transduced into cells with a multiplicity of infection of 20 . Then, the stable cell lines were purified.

\section{Immunohistochemistry (IHC)}

The slices were first dewaxed in xylene and then rehydrated in an alcohol gradient of $100 \%, 95 \%$, $85 \%$, and $75 \%$. Antigen retrieval was carried out by heating the slides after dipping them in a sodium citrate solution $(0.01 \mathrm{M}, \mathrm{pH} 6.0)$ at $95^{\circ} \mathrm{C}$ for $30 \mathrm{~min}$. Endogenous peroxidase activity was blocked by $3 \% \mathrm{H}_{2} \mathrm{O}_{2}$ for $10 \mathrm{~min}$ at room temperature. The samples were covered with $10 \%$ normal goat serum in phosphate buffered saline (PBS) for approximately $10 \mathrm{~min}$ at room temperature and then incubated with anti-KLF4 (rabbit polyclonal; Santa Cruz Biotechnology), anti-Cav-1 (mouse monoclonal; BD Biosciences), anti-Ecadherin (mouse monoclonal; Santa Cruz Biotechnology), and anti-vimentin (mouse monoclonal; Gene Tech Biotechnology) antibodies, respectively, at $4^{\circ} \mathrm{C}$ overnight. The slides were subsequently rinsed with PBS and incubated with peroxidase-conjugated secondary antibodies for $1 \mathrm{~h}$ at room temperature. The sections were washed using PBS and stained with diaminobenzidine for $5 \mathrm{~min}$. The specimens were counterstained with hematoxylin after rinsing, and the slides were dehydrated. Finally, the slides were mounted with coverslips and prepared for microscopic examination.

\section{IHC score}

Staining scores were generated by 2 independent observers blinded to the clinical data on the TMA. The staining scores consist of 2 indicators: staining intensity and proportion of positive cells. Staining intensity was classified into 4 grades: no staining (0), yellow (1), yellow-brown (2), and dark brown (3). The proportion of positive-staining cells was classified into 5 groups: $<10 \%$, marked $0 ; 10-25 \%$, marked 1 ; $25-50 \%$, marked $2 ; 50-75 \%$, marked 3 ; and $>75 \%$, marked 4 . The total score was equal to the product of 
Zhu et al.: Krüppel-Like Factor 4 Inhibits Epithelial-to-Mesenchymal Transition and Metastasis

the above 2 indicators. The total scores for KLF4, Cav-1, E-cadherin, and vimentin were divided relatively into 3 groups: negative, weak positive, and strong positive, as the total scores of $\leq 3,>3$ but $\leq 6$, and $>6$ were defined as negative, weak positive, and strong positive, respectively.

\section{Luciferase reporter assay}

The full-length promoter sequence of Cav-1 was constructed and the final full-length wild-type reporter plasmid (pLuc-Cav-1-wild) generated, which contained 7 KLF4/Cav-1-binding sites. The 7 binding sites in the Cav-1 promoter, including a putative KLF4-binding motif, were all mutated, and the mutated promoter (pGL4.27-Cav-1-mut) was synthesized. The plasmids were confirmed by DNA sequencing. To determine the transcriptional regulation of Cav-1 by KLF4, pLuc-Cav-1-wild or pGL4.27-Cav-1-mut were co-transfected into AsPC-1 cells with shKLF4 or control (50 nM). In addition, pLuc-Cav-1-wild or pGL4.27-Cav-1-mut were transfected into AsPC-1 cells with pcDNA3.1 (0.8 $\mu \mathrm{g})$ or pcDNA3.1 $(0.4 \mu \mathrm{g})$ and pKLF4 (0.4 $\mu \mathrm{g})$ or pKLF4 $(0.8 \mu \mathrm{g})$, respectively. Then, luciferase activity was detected using a luciferase reporter assay kit at $48 \mathrm{~h}$ after transfection. The activity of the Cav-1 promoter was normalized by the ratio of firefly luciferase activity to Renilla luciferase activity.

\section{Animal experiments}

Male athymic nude mice were purchased from Shanghai SLAC Laboratory Animal Community. The animals were maintained in the Animal Care Facility of Shanghai General Hospital. The stable AsPC-1 cell lines with shKLF4, control, and mock $\left(1.0 \times 10^{6}\right.$ cells in $0.1 \mathrm{~mL}$ Hank's balanced salt solution $)$ were injected subcutaneously into the right scapular region of nude mice. All mice were sacrificed at 21 days after injection or when they were on the verge of death, and tumor samples were harvested and weighed. A liver metastasis animal model was constructed with AsPC-1 cells $\left(1.0 \times 10^{6}\right.$ cells per mouse), which were injected intravenously into the superior mesenteric vein (SMV). Livers were removed from mice sacrificed at 35 days after injection or on the verge of death. After dissection into individual lobes, liver surface metastasis was counted by 2 independent researchers who were blinded to the experimental treatment. Then, liver metastasis tissues were examined by hematoxylin and eosin (H\&E) staining. The animal experiments were carried out according to the Shanghai General Hospital Animal Care and Use Guidelines, and the experimental protocols were approved by the Shanghai Resource Center of Laboratory Animals of the Chinese Academy of Science. All possible efforts were made to minimize animal suffering.

\section{Statistical analysis}

The significance in the correlation between the expression of KLF4/Cav-1/E-cadherin/vimentin and the clinicopathological parameters from the TMA specimens was determined by the Wilcoxon rank sum test. The association between KLF4 and Cav-1 expression was analyzed by pearman's test (r, $P$ ). In all tests, $P$ $<0.05$ was considered to be statistically significant. SPSS 22.0 software was used for all statistical analyses.

\section{Results}

KLF4 expression is down-regulated in pancreatic cancer tissues compared with normal pancreatic tissues in the Oncomine and The Cancer Genome Atlas (TCGA) databases

First, we browsed the Oncomine database and found that the expression of KLF4 mRNA was decreased in pancreatic adenocarcinoma (Fig. 1A). KLF4 expression was downregulated in pancreatic ductal adenocarcinoma compared with that in adjacent normal pancreas according to TCGA (Fig. 1B1). Further, data in TCGA showed that KLF4 expression was decreased in T4 compared with that in T1 and T2 (Fig. 1B2), and in stages IIB, III, and IV compared with that in stages IA, IB, and IIA (Fig. 1B3).

KLF4 protein expression is lower in pancreatic cancer tissues compared with normal tissues, and Cav-1 expression is higher in pancreatic cancer specimens compared with normal specimens; both proteins are closely related to the pathological features of pancreatic cancer

The expression of KLF4 and Cav-1 protein was initially detected in the TMA. KLF4, mainly in the nuclear compartment and cytoplasm, gave strong staining in normal tissue, with negative or weakly positive staining in cancer tissue (Fig. 2A). The low expression of KLF4 
Zhu et al.: Krüppel-Like Factor 4 Inhibits Epithelial-to-Mesenchymal Transition and Metastasis

Fig. 1. KLF4 expression is down-regulated in pancreatic cancer tissues compared with normal pancreatic tissues in the Oncomine and TCGA databases. A, The Oncomine database showed that KLF4 expression was decreased in pancreatic adenocarcinoma (Grützmann R, 2004) (fold change $=0.658, \mathrm{P}<0.05$ ). $\mathrm{B}, \mathrm{KLF} 4$ expression in pancreatic cancer in TCGA. B1, KLF4 expression was downregulated in pancreatic ductal adenocarcinoma $(\log 2$ copy number $=-0.024, \mathrm{P}<0.05) . \mathrm{B} 2, \mathrm{KLF} 4$ expression was decreased in T4 compared with T1 and $\mathrm{T} 2(\log 2$ copy number $=-0.029, \mathrm{P}<0.05)$. B3, KLF4 expression was down-regulated in stages IIB, III, and IV compared with stages I and IIA ( $\log 2$ copy number $=-0.032,-0.029,-0.036$, respectively, $\mathrm{P}<0.05$ ).

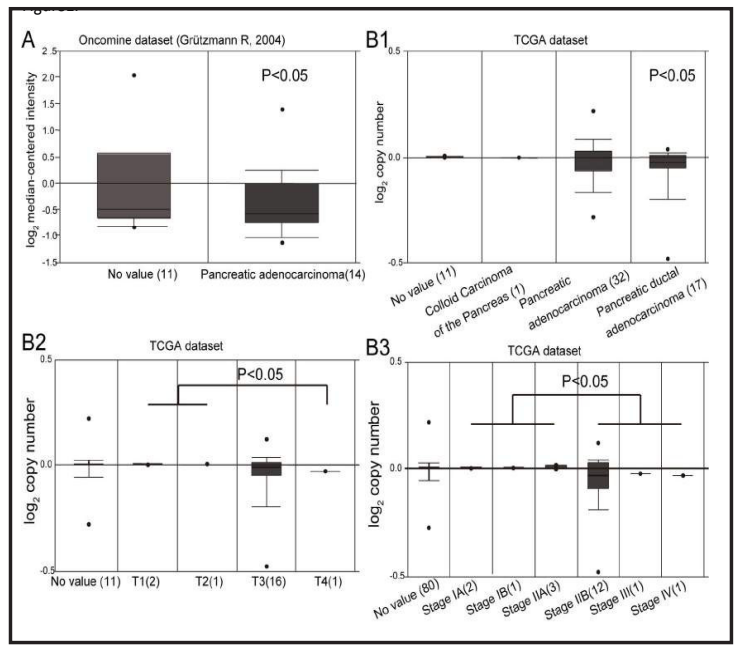

was correlated with poor tumor cell differentiation, and a significant difference in expression between grades I and II versus grade III was found (Fig. 2B). KLF4 expression was significantly lower in stages III and IV than in stages I and II, and the low expression of KLF4 was positively correlated with aggressive disease stages (Fig. 2C). In addition, KLF4 expression was negatively associated with tumor metastasis, as KLF4 expression was remarkably lower in samples with distant organ or lymph node metastasis compared with tissues without metastasis (Fig. 2D). These findings demonstrated that KLF4 plays a key role in the progression of pancreatic cancer and is a potential biomarker for this disease.

Cav-1 staining was mainly detected in the membrane and cytoplasm of tumor cells, and Cav-1 expression was significantly higher in pancreatic cancer samples than in adjacent normal tissues (Fig. 2E). IHC scores of Cav-1 were markedly higher in grade III than in grades I and II (Fig. 2F), as well as being markedly higher in stages III and IV than in stages I and II (Fig. 2G). In addition, Cav-1 expression was positively correlated with distant organ or lymph node metastasis (Fig. 2H). These results showed that Cav-1 may function as an oncogenic gene in pancreatic cancer.

E-cadherin expression is down-regulated in pancreatic cancer specimens compared to normal specimens, and vimentin expression is increased in pancreatic cancer specimens compared to normal specimens; both proteins are closely involved in the pathological features of pancreatic cancer

The expression of E-cadherin and vimentin protein was also detected in the TMA. E-cadherin was mainly located in the cell membrane and cytoplasm. Our study showed that E-cadherin staining was strongly positive in normal pancreatic tissue, whereas its staining in pancreatic cancer tissue was weakly positive or negative (Fig. 3A). The low expression of E-cadherin was observed more often in grade III pancreatic cancer than in grades I and II (Fig. 3B). Similarly, E-cadherin expression was negative correlated with aggressive disease stages (Fig. 3C) and distant organ or lymph node metastasis (Fig. 3D).

Vimentin staining was mainly detected in the cytoplasm of tumor cells, and vimentin expression in pancreatic cancer samples was evidently higher than that in adjacent normal tissues (Fig. 3E). The IHC scores of vimentin were markedly higher in grade III than in grades I and II (Fig. 3F), as well being markedly higher in stages III and IV than in stages I and II (Fig. 3G). Vimentin expression was positively correlated with distant organ or lymph node metastasis (Fig. 3H).

KLF4 and Cav-1 expression is closely associated with E-cadherin and vimentin expression in pancreatic cancer

To determine the relations between KLF4 and Cav-1 expression with E-cadherin and vimentin expression, we analyzed their expression using IHC. We found 41 cases that 
Zhu et al:: Krüppel-Like Factor 4 Inhibits Epithelial-to-Mesenchymal Transition and Metastasis

Fig. 2. KLF4 protein expression is lower in pancreatic cancer tissues compared with normal pancreatic tissues, and Cav-1 expression is higher in pancreatic cancer specimens compared with normal specimens. Both proteins are closely associated with the pathological features of pancreatic cancer. The TMA was stained immunohistochemically with specific anti-KLF4 and anti-Cav-1 antibodies. A, Representative images of KLF4 protein expression in normal pancreatic tissue and pancreatic cancer tissue are shown $(\times 200, \times 400)$. Note that most of the normal pancreatic tissues showed positive immunostaining for KLF4, whereas the great majority of pancreatic cancer tissues showed negative immunostaining. B, KLF4 expression was negatively correlated with tumor differentiation $(\mathrm{P}<0.05)$; representative images of grade II and III tumors are shown $(\times 200)$. C, KLF4 expression was negatively correlated with tumor stage $(\mathrm{P}<0.05)$; representative images of stage I and IV tumors are shown $(\times 200)$. D, KLF4 expression was negatively correlated with tumor metastasis $(\mathrm{P}<0.05)$; representative images of

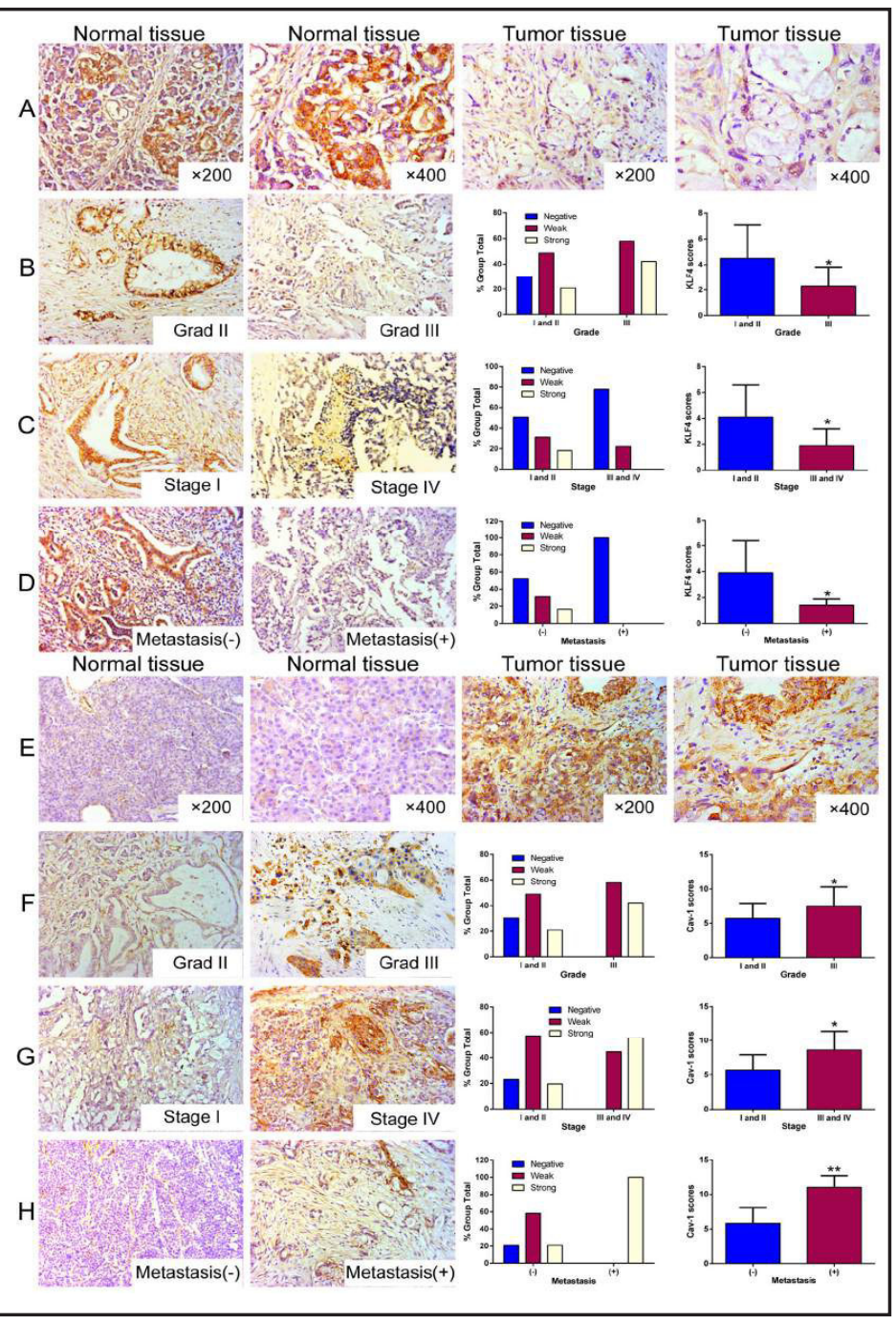
tumors with or without metastasis are shown ( $\times 200)$. E, Representative images of Cav-1 protein expression in normal pancreatic tissues and pancreatic cancer tissues are shown $(\times 200, \times 400)$. Note that most of the normal pancreatic tissues showed negative immunostaining for Cav-1, whereas a large number of pancreatic cancer tissues showed the opposite staining pattern. F, Cav-1 expression was positively correlated with tumor differentiation $(\mathrm{P}<0.05)$; representative images of grade II and III tumors are shown $(\times 200)$. G, Cav-1 expression was positively correlated with tumor stage $(\mathrm{P}<0.05)$; representative images of stage I and IV tumors are shown $(\times 200)$. H, Cav-1 expression was positively correlated with tumor metastasis $(\mathrm{P}<0.05)$; representative images of tumors with or without distant metastasis are shown $(\times 200)$.

were negative for KLF4 and E-cadherin staining and 5 cases that were positive for KLF4 and E-cadherin staining (Fig. 4A), which suggested a positive correlation between KLF4 and E-cadherin expression $(\mathrm{r}=0.341, P<0.01)$. Moreover, most of the KLF4-negative samples exhibited positive vimentin staining, which showed a negative correlation between both factors ( $\mathrm{r}=-0.468, P<0.01$, Fig. $4 \mathrm{~A}$ ). A great deal of pancreatic cancer samples with positive Cav-1 staining showed positive vimentin staining and negative E-cadherin staining, while negative Cav-1 staining samples exhibited negative vimentin staining and positive E-cadherin staining. These results indicated that Cav-1 was positively correlated with vimentin expression $(r=0.599, P<0.01$, Fig. $4 \mathrm{~B})$ and negatively correlated with E-cadherin expression ( $\mathrm{r}=-0.238, P<0.01$, Fig. 4B). These data suggested that KLF4 and Cav-1 may

\section{KARGER}


Fig. 3. E-cadherin expression is down-regulated in pancreatic cancer specimens compared with normal specimens, and vimentin expression is increased in pancreatic cancer specimens compared with normal specimens. Both proteins are closely associated with the pathological features of pancreatic cancer. A, Representative images of E-cadherin protein expression in normal pancreatic tissues and pancreatic cancer tissues are shown $(\times 200, \times 400)$. Note that most of the normal pancreatic tissues showed positive immunostaining for E-cadherin, whereas a large number of pancreatic cancer tissues showed negative staining. B, E-cadherin expression was negatively correlated with tumor differentiation $(\mathrm{P}<0.05)$; representative images of grade II and III tumors are shown $(\times 200)$. C, E-cadherin expression was negatively correlated with tumor stage $(\mathrm{P}<0.05)$; representative images of stage II and IV tumors are shown $(\times 200)$. D, E-cadherin expression was negatively correlated with tumor metastasis $(\mathrm{P}<0.05)$; representative images of tumors with

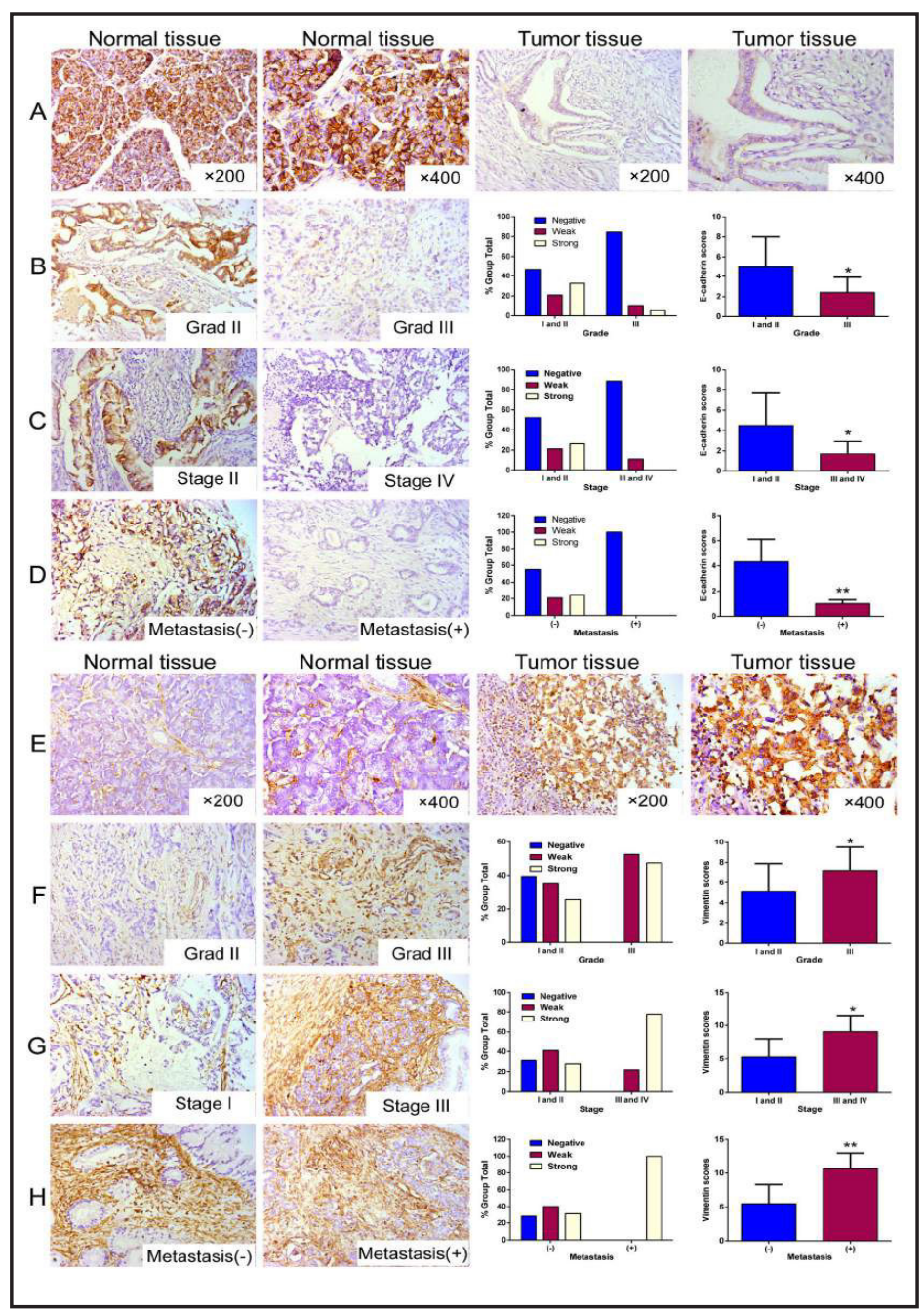
or without distant metastasis are shown $(\times 200)$. E, Representative images of vimentin protein expression in normal pancreatic tissues and pancreatic cancer tissues are shown $(\times 200, \times 400)$. Note that negative immunostaining for vimentin was found in the majority of normal tissues, whereas positive staining was observed in pancreatic cancer tissues. F, Vimentin expression was positively correlated with tumor differentiation $(\mathrm{P}<0.05)$; representative images of grade II and III tumors are shown $(\times 200)$. G, Vimentin expression was positively correlated with tumor stage $(\mathrm{P}<0.05)$; representative images of stage I and III tumors are shown $(\times 200)$. H, Vimentin expression was positively correlated with tumor metastasis $(\mathrm{P}<0.05)$; representative images of tumors with or without distant metastasis are shown $(\times 200)$.

be implicated in EMT by regulating E-cadherin and vimentin expression, which affects pancreatic cancer invasion and metastasis.

Down-regulating KLF4 expression promotes EMT and the motility of pancreatic cancer cells in vitro

To investigate the effect of altered KLF4 expression on EMT and metastasis of pancreatic cancer cells, we transduced AsPC-1 cells with shKLF4 for $48 \mathrm{~h}$. We observed that decreased KLF4 expression caused the typical transition from epithelial morphology to mesenchymal morphology (Fig. 5A). In addition, the cells were scratched with a $20-\mu \mathrm{L}$ pipette tip and incubated at $37^{\circ} \mathrm{C}$ for another $12 \mathrm{~h}$. The down-regulation of KLF4 expression facilitated the flattening and spreading of AsPC-1 cells (Fig. 5B). In cell migration and invasion assays, knockdown of KLF4 expression increased the number of cells permeating from the membrane 
Zhu et al.: Krüppel-Like Factor 4 Inhibits Epithelial-to-Mesenchymal Transition and Metastasis

Fig. 4. KLF4 and Cav-1 expression is closely associated with E-cadherin and vimentin expression in pancreatic cancer. A, Representative images of positive KLF4 and E-cadherin staining and negative vimentin staining (A1), and negative KLF4 and E-cadherin staining and positive vimentin staining (A2) in pancreatic cancer are shown $(\times 200, \times 400)$. Direct correlation between KLF4 and Ecadherin expression in pancreatic cancer samples was positive $(\mathrm{n}=$ 70; Pearson's correlation test, $\mathrm{r}=$ $0.341, \mathrm{P}<0.01$ ), while the correlation between KLF4 and vimentin expression was the opposite. $(\mathrm{r}=$

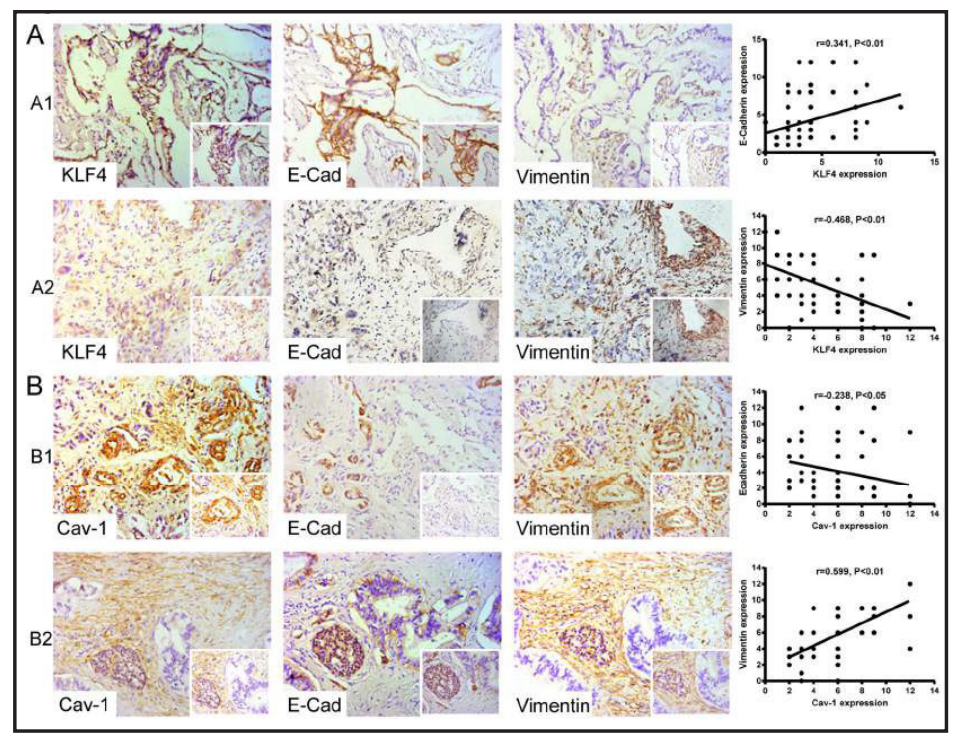
$-0.468, \mathrm{P}<0.01)$. Some of the dots on the graphs represent more than one sample (overlapped scores). B, Representative images of positive Cav-1 and vimentin staining and negative E-cadherin staining (B1), and negative Cav-1 and vimentin staining and positive E-cadherin staining (B2) are shown $(\times 200, \times 400)$. Direct correlation between Cav-1 and E-cadherin expression in pancreatic samples was negative $(\mathrm{r}=-0.238, \mathrm{P}<0.05)$, while the correlation between KLF4 and vimentin expression was the opposite $(r=0.599, \mathrm{P}<0.01)$. Some of the dots on the graphs represent more than one sample.

(Fig. 5C, D), indicating that down-regulating KLF4 expression increased the ability of AsPC-1 cells to undergo migration and invasion. Consequently, our findings clearly confirmed that KLF4 is an anti-oncogene and inhibits EMT and the migration and invasion of pancreatic cancer cells.

Down-regulating KLF4 expression promotes the growth and metastasis of pancreatic cancer cells in vivo

To determine the effect of altered KLF4 expression on the growth and metastasis of pancreatic cancer cells in vivo, stable AsPC-1 cell lines with shKLF4, control, and mock were injected subcutaneously into the right inguinal region $\left(1.0 \times 10^{6}\right.$ cells/mouse). The weight of tumors taken from shKLF4-nude mice was statistically higher than that of tumors from the other 2 groups after 21 days (Fig. 5E, F). In addition, these 3 stable cell lines were injected intravenously into the SMV $\left(1.0 \times 10^{6}\right.$ cells/mouse $)$ of nude mice. Consistently, the number of liver metastasis nodes in shKLF4-nude mice was markedly more than in the mock and control groups on day 35 (Fig. 5G, H). H\&E staining of metastatic liver tissues is shown in Fig. 5I. These data further demonstrated that KLF4 plays an anti-oncogenic role in the growth and metastasis of pancreatic cancer.

\section{KLF4 expression is negatively correlated with Cav-1 expression}

To explore how KLF4 participates in pancreatic cancer EMT and metastasis, we examined KLF4 and Cav-1 expression in the TMA. Among the primary pancreatic cancer tissue samples, we found 34 cases with negative KLF4 expression and positive Cav-1 expression. In addition, 10 cases were found with positive KLF4 expression and negative Cav-1 expression (Fig. 6A). These results indicated that there was a negative correlation between KLF4 and Cav-1 expression in pancreatic cancer $(r=-0.419, P<0.001$, Fig. 6B). Furthermore, western blot analysis of 5 paired pancreatic cancer tissue samples and adjacent normal pancreatic tissue samples also demonstrated a negative correlation between KLF4 and Cav-1 expression (Fig. 6C). 
Zhu et al:: Krüppel-Like Factor 4 Inhibits Epithelial-to-Mesenchymal Transition and Metastasis

Fig. 5. Down-regulating KLF4 expression promotes EMT and the motility of pancreatic cancer cells in vitro, and promotes the growth and metastasis of pancreatic cancer cells in vivo. AsPC-1 cells were transduced with shKLF4, control, or mock, respectively. A, Morphology transition from typical epithelial morphology to mesenchymal morphology. B, For the cell scratchwound assay, cell cultures were photographed and cell horizontal migration was assessed by measuring gap sizes. A smaller gap was shown after $12 \mathrm{~h}$ in AsPC-1 cells transfected with shKLF4. C, For the cell migration assay, the cells were maintained at $37^{\circ} \mathrm{C}$ for an additional $16 \mathrm{~h}$. The number of cells in 5 random fields in the lower surface that migrated through the membrane gap without Matrigel

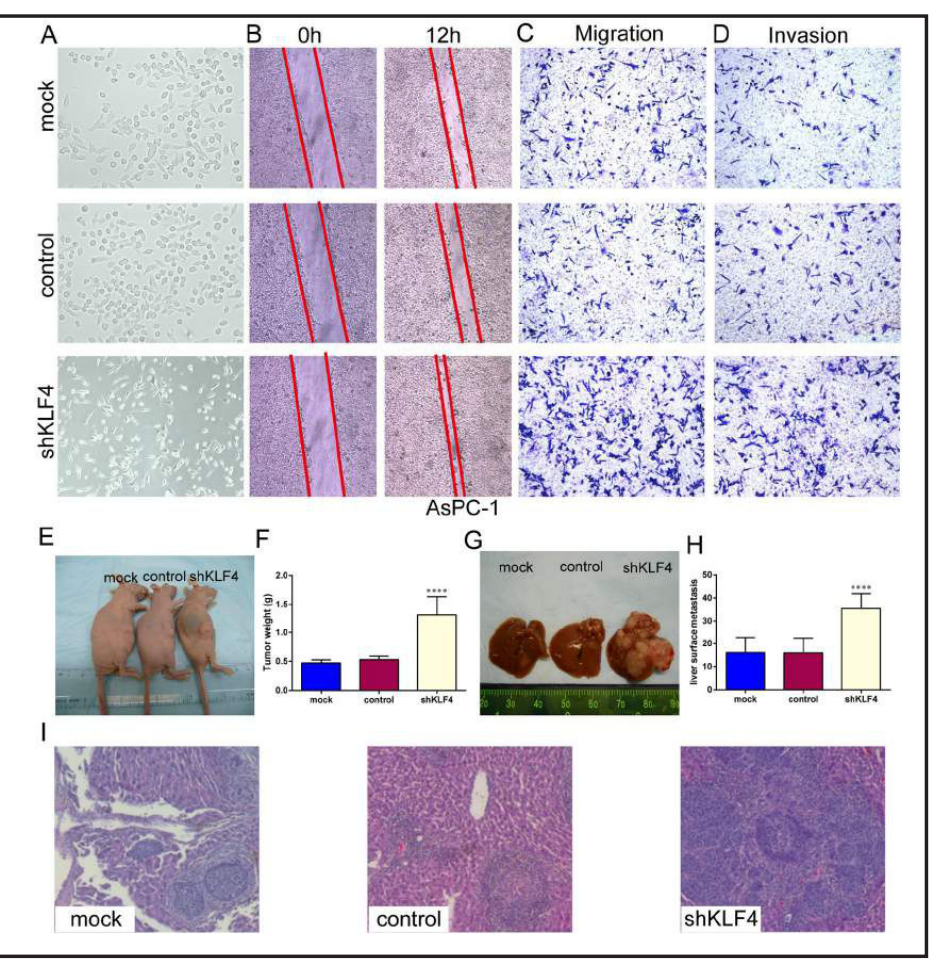
was counted. D, For the cell invasion assay, the cells were maintained at $37^{\circ} \mathrm{C}$ for an additional $24 \mathrm{~h}$. The number of cells in 5 random fields in the lower surface that penetrated through the membrane gap coated with Matrigel was counted. More cells were observed in the migration and invasion assays for the cells transfected with shKLF4 than in the control and mock groups. The stable AsPC-1 cell lines with shKLF4, control, and mock were respectively injected subcutaneously into the right inguinal region of 6 nude mice $\left(1.0 \times 10^{6}\right.$ cells $/$ mouse). The mice were sacrificed at 21 days after injection or when they were on the verge of death. These 3 stable cell lines were also injected intravenously into the SMV of 6 nude mice $\left(1.0 \times 10^{6}\right.$ cells/mouse). Livers were removed from the mice at 35 days after injection or when they were on the verge of death. Gross tumors in mice (E), liver metastasis tumors in the liver (G), and H\&E-stained sections of metastatic liver tissues (I) are depicted. Tumor weight $(\mathrm{F})$ and the number of liver surface metastases $(\mathrm{H})$ are presented. Bars, standard error. ${ }^{*} \mathrm{P}<0.05$ vs. mock and control.

To determine the effect of altered KLF4 expression on Cav-1 expression, we performed a loss-of-function assay. shKLF4-mediated knockdown of KLF4 expression increased Cav1 expression at the mRNA and protein level (Fig. 6D). Meanwhile, down-regulating KLF4 expression decreased E-cadherin expression, but increased vimentin expression at the mRNA and protein level (Fig. 6D). Thus, all of these results indicated that KLF4 may regulate EMT and metastasis of pancreatic cancer by down-regulating the expression of Cav- 1 .

\section{KLF4 inhibits Cav-1 expression by binding directly to the Cav-1 promoter region}

To identify further the underlying mechanisms by which KLF4 down-regulates Cav-1 expression, we analyzed the Cav-1 promoter sequence for possible KLF4-binding sites, and confirmed the presence of 7 putative binding sites (Fig. 6E). A full-length Cav-1 promoter (pLuc-Cav-1-wild, containing all of the putative KLF4-binding sites) was generated. Then, we mutated all 7 of the binding sites (Fig. 6F) and synthesized a mutant promoter (pGL4.27Cav-1-mut). To determine whether KLF4 negatively regulated Cav-1 expression at the transcriptional level, pLuc-Cav-1-wild or pGL4.27-Cav-1-mut with shKLF4 or control (50 nM) were co-transfected into AsPC-1 cells. As shown in Fig. 6G, shKLF4 significantly increased the activity of the wild-type Cav-1 promoter, whereas it had no effect on the activity of the mutant Cav-1 promoter in AsPC-1 cells. Then, pLuc-Cav-1-wild or pGL4.27-Cav-1-mut was

\section{KARGER}


Fig. 6. KLF4 inhibits Cav-1 expression by binding directly to the Cav1 promoter region. A, Representative images of negative KLF4 and positive Cav-1 staining (A1) and positive KLF4 and negative Cav-1 staining (A2) in the TMA are shown $(\times 200, \times 400) . B$, Direct correlation between KLF4 and Cav-1 expression in pancreatic samples was negative ( $\mathrm{n}=70$; Pearson's correlation test, $\mathrm{r}=-0.419, \mathrm{P}<0.001)$. Some of the dots in the graphs represent more than one sample. C, Western blot analysis of KLF4 and Cav-1 protein expression in 5 paired pancreatic cancer and normal pancreatic tissue samples also indicated the negative correlation between KLF4 and Cav-1 expression. D, RT-PCR and western blot assays of KLF4, Cav-1, and EMT markers in AsPC-1 cells transfected with shKLF4. The results showed decreased KLF4 and E-cadherin expression and increased Cav-1 and vimentin expression at the mRNA and protein level after transfection with shKLF4. E, A schematic representation of the 7 binding sites in the Cav-1 promoter, including the putative KLF4-binding motif. F, The 7 binding sites were all mutated, and a schematic representation is

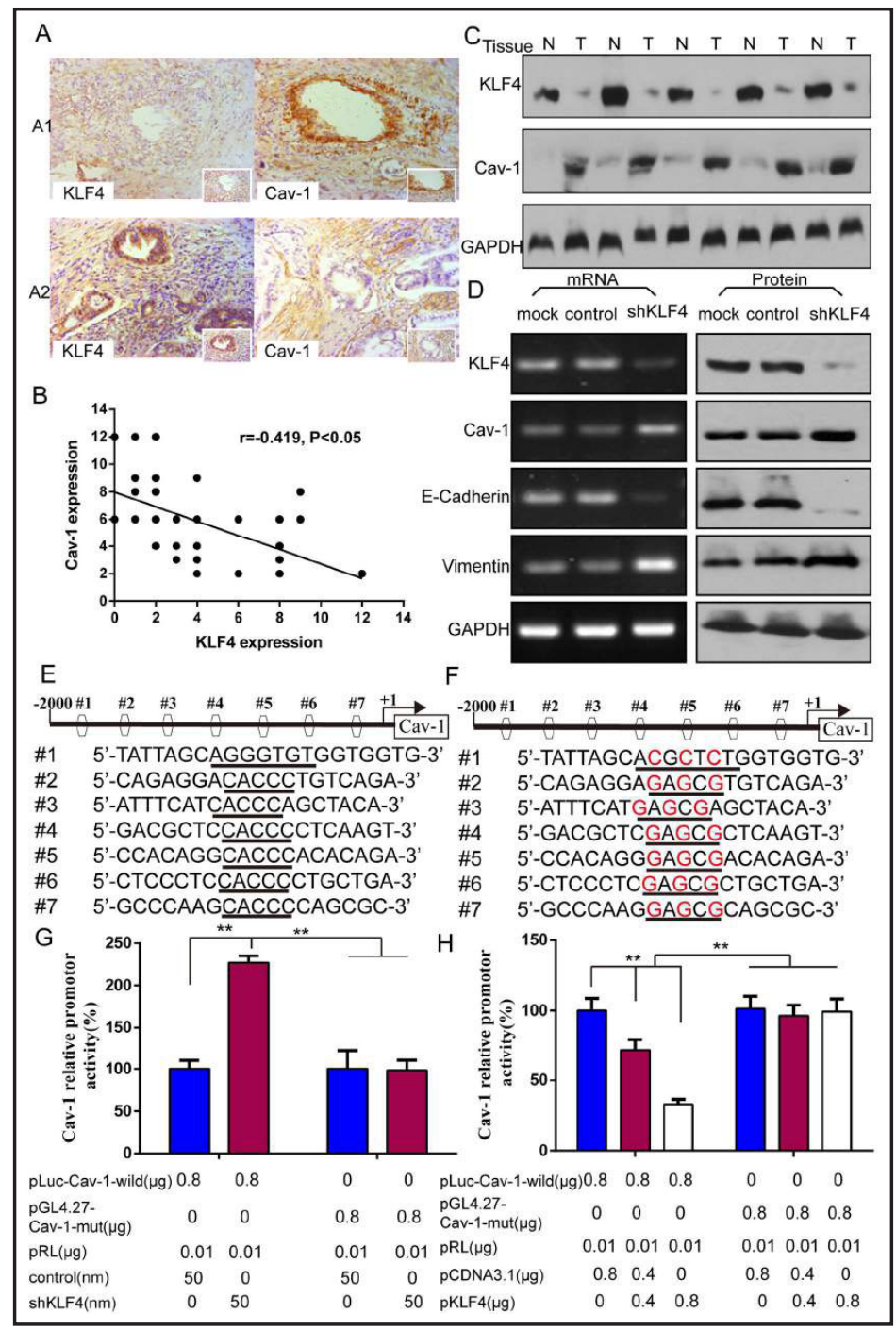
shown. G, The full-length wild-type Cav-1 promoter (pLuc-Cav-1-wild) or mutant promoter (pGL4.27-Cav-1-mut) was respectively transfected into AsPC-1 cells with shKLF4 or control. Cav-1 promoter activity was detected after $48 \mathrm{~h}$ using a luciferase reporter assay. The results are shown as \% of firefly luciferase activity vs. Renilla luciferase activity. H. pLucCav-1-wild or pGL4.27-Cav-1-mut was transfected into AsPC-1 cells with pcDNA3.1 (0.8 $\mu \mathrm{g}$ ) or pcDNA3.1 $(0.4 \mu \mathrm{g})$ and pKLF4 $(0.4 \mu \mathrm{g})$ or pKLF4 $(0.8 \mu \mathrm{g})$, respectively. Cav-1 promoter activity was detected after $48 \mathrm{~h}$ using a luciferase reporter assay. The results are shown as \% of firefly luciferase activity vs. Renilla luciferase activity. Bars standard error. ${ }^{*} \mathrm{P}<0.05$.

transfected into AsPC-1 cells with pcDNA3.1 $(0.8 \mu \mathrm{g})$ or pcDNA3.1 $(0.4 \mu \mathrm{g})$ and pKLF4 (0.4 $\mu \mathrm{g})$ or pKLF4 $(0.8 \mu \mathrm{g})$, respectively. pKLF4 $(0.8 \mu \mathrm{g})$ and pKLF4 $(0.4 \mu \mathrm{g})$ both significantly decreased the activity of the wild-type Cav-1 promoter, but did not decrease the activity of the mutant Cav-1 promoter (Fig. $6 \mathrm{H}$ ). Taken together, these results strongly suggested that KLF4 transcriptionally inhibits Cav- 1 expression by binding directly to the Cav-1 promoter domain.

\section{Discussion}

In this study, we investigated the crucial role of KLF4 and Cav-1 in the progression of pancreatic cancer and the possible underlying mechanisms. We showed that KLF4 and Cav-1 
were closely associated with the clinicopathological behavior of pancreatic cancer. In addition, both KLF4 and Cav-1 affected the expression of epithelial and mesenchymal morphology biomarkers, and KLF4 expression was negatively correlated with Cav-1 expression. Furthermore, we identified that KLF4 transcriptionally inhibited Cav-1 expression by binding to the Cav-1 promoter domain, establishing a novel KLF4/Cav-1 signaling pathway that regulates EMT and metastasis of pancreatic cancer. In summary, our clinical and experimental data demonstrated that down-regulating KLF4 expression increases Cav-1 expression and ultimately promotes EMT and metastasis of pancreatic cancer.

KLF4, a zinc finger-type transcription factor, has been demonstrated to participate in the transcriptional regulation of a variety of oncogenes and tumor suppressors [3335]. However, KLF4 plays a dual functional role in cancer development and progression, acting as an oncogene or anti-oncogene. For example, KLF4 acts as a tumor suppressor to suppress the cell migration and invasion of esophageal cancer [36]. In addition, Zhang et al. reported that KLF4 transcriptionally represses $\beta$-catenin expression and inhibits the proliferation, invasion, and metastasis of gastric cancer [37]. Moreover, KLF4 expression is markedly down-regulated in colon cancer, and the loss of KLF4 can serve as an independent predictor of its recurrence and survival [38]. However, KLF4 reportedly acts as an oncogene in melanoma and prostate cancer $[39,40]$. The dual function of KLF4 in cancer biological behavior may shift depending on tumor type, tumor stage, and research approach. In the current study, we listed several lines of evidence to prove that KLF4 is a tumor suppressor in pancreatic cancer. First, clinical data indicated that KLF4 was down-regulated in pancreatic cancer tissue compared with normal pancreatic tissue. Furthermore, KLF4 expression was negatively correlated with tumor differentiation, disease stage, and lymph node or distant organ metastasis. In addition, experimental data indicated that KLF4 knockdown promoted pancreatic cancer cell growth and metastasis in vitro and in vivo, indicating that KLF4 inhibits pancreatic cancer invasion and metastasis. Thus, our clinical and experimental data all identified KLF4 as a tumor suppressor in pancreatic cancer, which is in agreement with recent studies [17-21].

Mounting evidence indicates that EMT plays a critical role in cancer metastasis [4144]. In the present study, IHC results suggested that KLF4 expression is positively correlated with the expression of E-cadherin and negatively associated with the expression of vimentin. Moreover, KLF4 knockdown decreased E-cadherin expression and elevated vimentin expression in AsPC-1 cells. Specifically, decreased KLF4 expression promoted the transition from a typical epithelial morphology to a typical mesenchymal morphology in AsPC-1 cells. Our results were consistent with recent publications. For example, the overexpression of KLF4 increased E-cadherin expression, but decreased vimentin expression and subsequently inhibited EMT in lung cancer [45]. Also, KLF4 overexpression inhibited EMT in hepatocellular carcinoma by reducing the effects of other factors [46]. Taken together, we have clearly demonstrated that KLF4 can inhibit EMT in pancreatic cancer by increasing E-cadherin expression and decreasing vimentin expression.

In order to investigate the underlying mechanisms of KLF4 expression on pancreatic cancer progression, we explored its targets for downstream genes. Our study proved that Cav-1, an important constituent of caveolae and involved in multiple cell-signaling events, may be a new downstream target of KLF4. Further, our results showed that high levels of Cav-1 expression were associated with poorer tumor differentiation, progressive disease stage, and lymph node or distant organ metastasis. More importantly, KLF4 expression was negatively correlated with Cav-1 expression, as shown by IHC and western blot analyses. Additionally, knockdown of KLF4 expression elevated Cav-1 expression. Furthermore, bioinformatics analysis showed that there were 7 putative binding sites in the Cav-1 promoter, including a putative KLF4-binding motif. We constructed the full-length Cav-1 promoter and muted all 7 of these binding sites. A luciferase reporter assay demonstrated that KLF4 can bind directly to specific Cav-1 promoter elements and negatively regulate Cav-1 expression transcriptionally. Collectively, KLF4 may be involved in pancreatic cancer progression by negatively regulating Cav-1 expression. 


\section{Cellular Physiology Cell Physiol Biochem 2018;46:238-252 \\ \begin{tabular}{c|c|c|c|} 
DOI: 10.1159/000488426 & O 2018 The Author(s). Published by S. Karger AG, Basel \\
www.karger.com/cpb
\end{tabular} \\ Zhu et al.: Krüppel-Like Factor 4 Inhibits Epithelial-to-Mesenchymal Transition and Metastasis}

Cav-1 expression was negatively correlated with E-cadherin expression, but positively correlated with vimentin expression. Our previous research indicated that Cav-1 can regulate the expression of E-cadherin and then regulate EMT in pancreatic cancer $[32,47]$. We speculate that KLF4 may regulate EMT in pancreatic cancer by negatively regulating Cav-1 expression. However, some reports have shown that KLF4 can directly regulate the expression of E-cadherin and vimentin $[45,48]$. Thus, KLF4 may regulate EMT by directly regulating E-cadherin and vimentin expression. In summary, our study established a novel KLF4/Cav-1 signaling pathway and demonstrated its crucial role in the progression of pancreatic cancer.

It has been reported that KLF4 can transcriptionally regulate many other genes. In colon cancer, KLF4 protein can bind directly to the IFITM3 promoter and transcriptionally inhibit IFITM3 expression [49]. Li et al. demonstrated that KLF4 could negatively regulate FoxM1 expression in gastric cancer using luciferase reporter and chromatin immunoprecipitation assays [50]. Recently, KLF4 was reported to trans-activate HNF-6 expression-induced tumor cell differentiation and repress hepatocellular carcinoma invasion and migration [51]. In addition, KLF4 acts as an anti-oncogene in lung cancer by inhibiting hTERT expression and telomerase activity [52]. However, KLF4 may also be regulated by some other factors. In gastric cancer, miR-32, acting as an oncogenic microRNA, was shown to bind to the $3^{\prime}$-untranslated region of KLF4 mRNA and inhibit KLF4 mRNA translation for protein production [53]. In addition, miR-152 can regulate KLF4 expression in the same manner in glioblastoma [54]. Thus, KLF4 is involved in various regulatory mechanisms, but the crosstalk between these pathways has yet to be identified in pancreatic cancer.

\section{Conclusion}

In summary, the present study has not only presented critical insights into the role of KLF4 in the progression of pancreatic cancer but also uncovered a correlation between KLF4 and Cav-1 expression. Furthermore, a novel KLF4/Cav-1 signaling pathway in EMT and metastasis was identified. Collectively, our results have identified an original molecular mechanism for KLF4 in the progression of pancreatic cancer, and revealed that dysregulated KLF4/Cav-1 signaling is a prospective molecular target for the design of novel therapeutic methods to prevent the progression of pancreatic cancer. However, deeper investigations are needed to elaborate further the dysregulation and biological roles of KLF4 in pancreatic cancer.

\section{Abbreviations}

KLF (Krüppel-like factor); Cav-1 (Caveolin-1); EMT (Epithelial-to-mesenchymal transition); TMA (Tissue microarray); IHC (Immunohistochemistry); PBS (Phosphate buffered saline); SMV (Superior mesenteric vein); H\&E (Hematoxylin and eosin); TCGA (The Cancer Genome Atlas).

\section{Acknowledgements}

The use of tissue samples accorded with the relevant guidelines and regulations, and ethics approval was granted by the Shanghai General Hospital Institutional Review Board. Animal experiments were carried out according to the Shanghai General Hospital Animal Care and Use Guidelines, and the experimental protocols were approved by the Shanghai Resource Center of Laboratory Animals of the Chinese Academy of Science.

This research was supported by grants from the National Natural Science Foundation of China (817725276), Shanghai Municipal Education Commission-Gaofeng Clinical Medicine 
Zhu et al.: Krüppel-Like Factor 4 Inhibits Epithelial-to-Mesenchymal Transition and Metastasis

Grant Support (20161425), Shanghai Jiaotong University Medical Cross Fund (YG2017MS28), Shanghai Municipal Science and Technology Committee (14411966800), and the Techpool Fund (UF201419).

C. H. and Z. Z. designed the experiments, analyzed the data, and revised the manuscript. Z. Y., L. Z., and J. W. performed most of the experiments and wrote the manuscript. J. Z., B. Y., J. D., and Z. Q. performed the experiments. All of the authors discussed the results and reviewed the manuscript.

\section{Disclosure Statement}

The authors declare no competing financial interests.

\section{References}

1 Torre LA, Bray F, Siegel RL, Ferlay J, Lortet-Tieulent J, Jemal A: Global cancer statistics, 2012 CA Cancer J Clin 2015;65:87-108.

2 Siegel RL, Miller KD, Jemal A: Cancer Statistics, 2017 CA Cancer J Clin 2017;67:7-30.

- 3 Chen W, Zheng R, Baade PD, Zhang S, Zeng H, Bray F, Jemal A, Yu XQ He J: Cancer statistics in China, 2015. CA Cancer J Clin 2016;66:115-132.

4 Paulson AS, Tran Cao HS, Tempero MA, Lowy AM: Therapeutic advances in pancreatic cancer. Gastroenterology 2013;144:1316-1326.

5 Huang C, Xie D, Cui J, Li Q Gao Y, Xie K: FOXM1c promotes pancreatic cancer epithelial-to-mesenchymal transition and metastasis via upregulation of expression of the urokinase plasminogen activator system. Clin Cancer Res 2014;20:1477-1488.

6 Preiss A, Rosenberg UB, Kienlin A, Seifert E, Jackle H: Molecular genetics of Kruppel, a gene required for segmentation of the Drosophila embryo. Nature 1985;313:27-32.

7 Ghaleb AM, Laroui H, Merlin D, Yang VW: Genetic deletion of Klf4 in the mouse intestinal epithelium ameliorates dextran sodium sulfate-induced colitis by modulating the NF-kappaB pathway inflammatory response. Inflamm Bowel Dis 2014;20:811-820.

8 Farrugia MK, Vanderbilt DB, Salkeni MA, Ruppert JM: Kruppel-like Pluripotency Factors as Modulators of Cancer Cell Therapeutic Responses. Cancer Res 2016;76:1677-1682.

-9 Liang S, Yu H, Chen X, Shen T, Cui Z, Si G, Zhang J, Cheng Y, Jia S, Song S, Zhang X, Yu X: PDGF-BB/KLF4/VEGF Signaling Axis in Pulmonary Artery Endothelial Cell Angiogenesis. Cell Physiol Biochem 2017;41:23332349.

10 Zhu LF, Chen QR, Chen SZ, Wang LY, Luo XF, Ren JH, Yuan XH, Wu XQ, Zeng YL, Xiao M, Chen YQ, Chen YY, Lin MH, Wu ZJ, Chen ZZ, Hu JD, Yang T: The Construction and Identification of Induced Pluripotent Stem Cells Derived from Acute Myelogenous Leukemia Cells. Cell Physiol Biochem 2017;41:1661-1674.

11 Ferralli J, Chiquet-Ehrismann R, Degen M: KLF4alpha stimulates breast cancer cell proliferation by acting as a KLF4 antagonist. Oncotarget 2016;7:45608-45621.

12 Yu T, Chen X, Lin T, Liu J, Li M, Zhang W, Xu X, Zhao W, Liu M, Napier DL, Wang C, Evers BM, Liu C: KLF4 deletion alters gastric cell lineage and induces MUC2 expression. Cell Death Dis 2016;7:e2255.

13 Yang Y, Katz JP: KLF4 is downregulated but not mutated during human esophageal squamous cell carcinogenesis and has tumor stage-specific functions. Cancer Biol Ther 2016;17:422-429.

14 Huang J, Liu K, Song D, Ding M, Wang J, Jin Q, Ni J: Kruppel-like factor 4 promotes high-mobility group box 1-induced chemotherapy resistance in osteosarcoma cells. Cancer Sci 2016;107:242-249.

15 Le Magnen C, Bubendorf L, Ruiz C, Zlobec I, Bachmann A, Heberer M, Spagnoli GC, Wyler S, Mengus C: Klf4 transcription factor is expressed in the cytoplasm of prostate cancer cells. Eur J Cancer 2013;49:955-963.

16 Wei D, Kanai M, Huang S, Xie K: Emerging role of KLF4 in human gastrointestinal cancer. Carcinogenesis 2006;27:23-31.

17 Shi M, Cui J, Du J, Wei D, Jia Z, Zhang J, Zhu Z, Gao Y, Xie K: A novel KLF4/LDHA signaling pathway regulates aerobic glycolysis in and progression of pancreatic cancer. Clin Cancer Res 2014;20:4370-4380. 


\section{Cellular Physiology Cell Physiol Biochem 2018;46:238-252

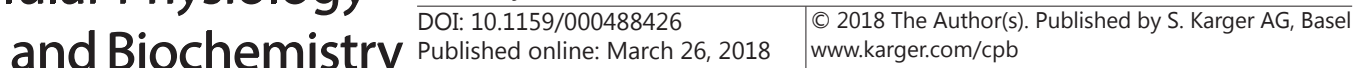 and Biochemistry $\frac{\text { Published online: Marke }}{\text { Zhu et al.: Krüppel-Like Factor } 4 \text { Inhibits Epithelial-to-Mesenchymal Transition and }}$}

Metastasis

18 Zammarchi F, Morelli M, Menicagli M, Di Cristofano C, Zavaglia K, Paolucci A, Campani D, Aretini P, Boggi U, Mosca F, Cavazzana A, Cartegni L, Bevilacqua G, Mazzanti CM: KLF4 is a novel candidate tumor suppressor gene in pancreatic ductal carcinoma. Am J Pathol 2011;178:361-372.

19 Wei D, Kanai M, Jia Z, Le X, Xie K: Kruppel-like factor 4 induces p27Kip1 expression in and suppresses the growth and metastasis of human pancreatic cancer cells. Cancer Res 2008;68:4631-4639.

20 Guo K, Cui J, Quan M, Xie D, Jia Z, Wei D, Wang L, Gao Y, Ma Q, Xie K: The Novel KLF4/MSI2 Signaling Pathway Regulates Growth and Metastasis of Pancreatic Cancer. Clin Cancer Res 2017;23:687-696.

21 Yan Y, Li Z, Kong X, Jia Z, Zuo X, Gagea M, Huang S, Wei D, Xie K: KLF4-Mediated Suppression of CD44 Signaling Negatively Impacts Pancreatic Cancer Stemness and Metastasis. Cancer Res 2016;76:2419-2431.

-22 Tiwari N, Meyer-Schaller N, Arnold P, Antoniadis H, Pachkov M, van Nimwegen E, Christofori G: Klf4 is a transcriptional regulator of genes critical for EMT, including Jnk1 (Mapk8). PLoS One 2013;8:e57329.

23 Huang C, Xie K: Crosstalk of Sp1 and Stat3 signaling in pancreatic cancer pathogenesis. Cytokine Growth Factor Rev 2012;23:25-35.

24 Huang C, Du J, Xie K: FOXM1 and its oncogenic signaling in pancreatic cancer pathogenesis. Biochim Biophys Acta 2014;1845:104-116.

25 Sun XJ, Liu BY, Yan S, Jiang TH, Cheng HQ, Jiang HS, Cao Y, Mao AW: MicroRNA-29a Promotes Pancreatic Cancer Growth by Inhibiting Tristetraprolin. Cell Physiol Biochem 2015;37:707-718.

26 Basu Roy UK, Henkhaus RS, Loupakis F, Cremolini C, Gerner EW, Ignatenko NA: Caveolin-1 is a novel regulator of K-RAS-dependent migration in colon carcinogenesis. Int J Cancer 2013;133:43-57.

-27 Kang J, Park JH, Lee HJ, Jo U, Park JK, Seo JH, Kim YH, Kim I, Park KH: Caveolin-1 Modulates DocetaxelInduced Cell Death in Breast Cancer Cell Subtypes through Different Mechanisms. Cancer Res Treat 2016;48:715-726.

-28 Yu F, Xie D, Ng SS, Lum CT, Cai MY, Cheung WK, Kung HF, Lin G, Wang X, Lin MC: IFITM1 promotes the metastasis of human colorectal cancer via CAV-1. Cancer Lett 2015;368:135-143.

29 Zhang PX, Cheng J, Zou S, D’Souza AD, Koff JL, Lu J, Lee PJ, Krause DS, Egan ME, Bruscia EM: Pharmacological modulation of the AKT/microRNA-199a-5p/CAV1 pathway ameliorates cystic fibrosis lung hyper-inflammation. Nat Commun 2015;6:6221.

- 30 Nam KH, Lee BL, Park JH, Kim J, Han N, Lee HE, Kim MA, Lee HS, Kim WH: Caveolin 1 expression correlates with poor prognosis and focal adhesion kinase expression in gastric cancer. Pathobiology 2013;80:87-94.

-31 Diaz-Valdivia N, Bravo D, Huerta H, Henriquez S, Gabler F, Vega M, Romero C, Calderon C, Owen GI, Leyton L, Quest AF: Enhanced caveolin-1 expression increases migration, anchorage-independent growth and invasion of endometrial adenocarcinoma cells. BMC Cancer 2015;15:463.

-32 Huang C, Qiu Z, Wang L, Peng Z, Jia Z, Logsdon CD, Le X, Wei D, Huang S, Xie K: A novel FoxM1-caveolin signaling pathway promotes pancreatic cancer invasion and metastasis. Cancer Res 2012;72:655-665.

-33 Ke B, Zhang A, Wu X, Fang X: The Role of Kruppel-like Factor 4 in Renal Fibrosis. Front Physiol 2015;6:327.

-34 Ding B, Liu P, Liu W, Sun P, Wang CL: Emerging roles of Kruppel-like factor 4 in cancer and cancer stem cells. Asian Pac J Cancer Prev 2015;16:3629-3633.

-35 Lu XJ, Shi Y, Chen JL, Ma S: Kruppel-like factors in hepatocellular carcinoma. Tumour Biol 2015;36:533-541.

-36 Tian Y, Luo A, Cai Y, Su Q, Ding F, Chen H, Liu Z: MicroRNA-10b promotes migration and invasion through KLF4 in human esophageal cancer cell lines. J Biol Chem 2010;285:7986-7994.

-37 Zhang N, Zhang J, Shuai L, Zha L, He M, Huang Z, Wang Z: Kruppel-like factor 4 negatively regulates betacatenin expression and inhibits the proliferation, invasion and metastasis of gastric cancer. Int J Oncol 2012;40:2038-2048.

-38 Patel NV, Ghaleb AM, Nandan MO, Yang VW: Expression of the tumor suppressor Kruppel-like factor 4 as a prognostic predictor for colon cancer. Cancer Epidemiol Biomarkers Prev 2010;19:2631-2638.

-39 Shin SH, Kwon YW, Heo SC, Jeong GO, Kim BR, Seo EJ, Kim JH: Kruppel-like factor 4 mediates lysophosphatidic acid-stimulated migration and proliferation of PC3M prostate cancer cells. Exp Mol Med 2014;46:e104.

40 Riverso M, Montagnani V, Stecca B: KLF4 is regulated by RAS/RAF/MEK/ERK signaling through E2F1 and promotes melanoma cell growth. Oncogene 2017;36:3322-3333.

41 Nieto MA, Huang RY, Jackson RA, Thiery JP: EMT: 2016. Cell 2016;166:21-45.

-42 Fischer KR, Durrans A, Lee S, Sheng J, Li F, Wong ST, Choi H, El Rayes T, Ryu S, Troeger J, Schwabe RF, Vahdat LT, Altorki NK, Mittal V, Gao D: Epithelial-to-mesenchymal transition is not required for lung metastasis but contributes to chemoresistance. Nature 2015;527:472-476. 


\section{Cellular Physiology Cell Physiol Biochem 2018;46:238-252

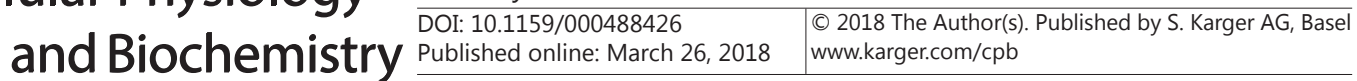 \\ Zhu et al.: Krüppel-Like Factor 4 Inhibits Epithelial-to-Mesenchymal Transition and Metastasis}

43 He M, Zhan M, Chen W, Xu S, Long M, Shen H, Shi Y, Liu Q, Mohan M, Wang J: MiR-143-5p Deficiency Triggers EMT and Metastasis by Targeting HIF-1alpha in Gallbladder Cancer. Cell Physiol Biochem 2017;42:2078-2092.

-44 Zhao L, Sun H, Kong H, Chen Z, Chen B, Zhou M: The Lncrna-TUG1/EZH2 Axis Promotes Pancreatic Cancer Cell Proliferation, Migration and EMT Phenotype Formation Through Sponging Mir-382. Cell Physiol Biochem 2017;42:2145-2158.

45 Liu S, Yang H, Chen Y, He B, Chen Q: Kruppel-Like Factor 4 Enhances Sensitivity of Cisplatin to Lung Cancer Cells and Inhibits Regulating Epithelial-to-Mesenchymal Transition. Oncol Res 2016;24:81-87.

-46 Li Q Song W, Wang W, Yao S, Tian C, Cai X, Wang L: Suppression of epithelial-mesenchymal transition in hepatocellular carcinoma cells by Kruppel-like factor 4. Oncotarget 2016;7:29749-29760.

47 Zhang K, Yang G, Wu W, Zhang J, Xia X, Jiang T, Cao J, Huang K, Qiu Z, Huang C: Decreased Expression of Caveolin-1 and E-Cadherin Correlates with the Clinicopathologic Features of Gastric Cancer and the EMT Process. Recent Pat Anticancer Drug Discov 2016;11:236-244.

-48 Yori JL, Johnson E, Zhou G, Jain MK, Keri RA: Kruppel-like factor 4 inhibits epithelial-to-mesenchymal transition through regulation of E-cadherin gene expression. J Biol Chem 2010;285:16854-16863.

49 Li D, Peng Z, Tang H, Wei P, Kong X, Yan D, Huang F, Li Q, Le X, Li Q, Xie K: KLF4-mediated negative regulation of IFITM3 expression plays a critical role in colon cancer pathogenesis. Clin Cancer Res 2011;17:3558-3568.

50 Li Q Jia Z, Wang L, Kong X, Li Q, Guo K, Tan D, Le X, Wei D, Huang S, Mishra L, Xie K: Disruption of Klf4 in villin-positive gastric progenitor cells promotes formation and progression of tumors of the antrum in mice. Gastroenterology 2012;142:531-542.

-51 Sun H, Tang H, Xie D, Jia Z, Ma Z, Wei D, Mishra L, Gao Y, Zheng S, Xie K, Peng Z: Kruppel-like Factor 4 Blocks Hepatocellular Carcinoma Dedifferentiation and Progression through Activation of Hepatocyte Nuclear Factor-6. Clin Cancer Res 2016;22:502-512.

52 Hu W, Jia Y, Xiao X, Lv K, Chen Y, Wang L, Luo X, Liu T, Li W, Li Y, Zhang C, Yu Z, Huang W, Sun B, Deng WG: KLF4 downregulates hTERT expression and telomerase activity to inhibit lung carcinoma growth. Oncotarget 2016;7:52870-52887.

53 Yan C, Yu J, Liu Y, Kang W, Ma Z, Zhou L: MiR-32 promotes gastric carcinoma tumorigenesis by targeting Kruppel-like factor 4. Biochem Biophys Res Commun 2015;467:913-920.

54 Ma J, Yao Y, Wang P, Liu Y, Zhao L, Li Z, Li Z, Xue Y: MiR-152 functions as a tumor suppressor in glioblastoma stem cells by targeting Kruppel-like factor 4. Cancer Lett 2014;355:85-95. 